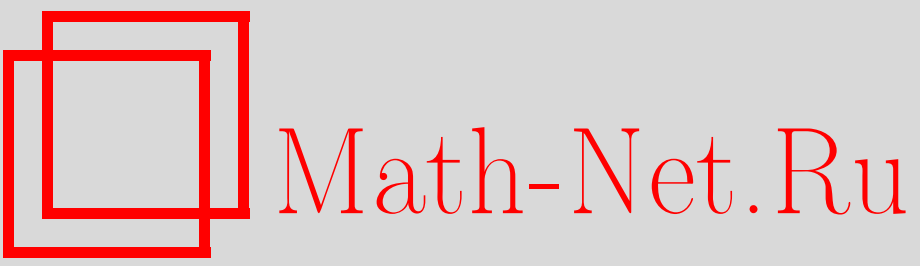

Ф. М. Мухамедов, У. А. Розиков, Экстремальность неупорядоченной фазы неоднородной модели Поттса на дереве Кэли, ТМФ, 2000, том 124, номер 3, 410-418

DOI: https://doi.org/10.4213/tmf647

Использование Общероссийского математического портала Math-Net.Ru подразумевает, что вы прочитали и согласны с пользовательским соглашением

http://www.mathnet.ru/rus/agreement

Параметры загрузки:

IP: 34.227 .88 .159

26 апреля 2023 г., 12:26:47 
ТЕОРЕТИЧЕСКАЯ

И МАТЕМАТИЧЕСКАЯ

ФИЗИКА

Том 124, № 3

сентябрь, 2000

(C) $\mathbf{2 0 0 0}$ г.

Ф.М. Мухамедов* , У.А. Розиков*

\section{ЭКСТРЕМАЛЬНОСТЬ НЕУПОРЯДОЧЕННОЙ ФАЗЫ НЕОДНОРОДНОЙ МОДЕЛИ ПОТТСА НА ДЕРЕВЕ КЭЛИ}

Найдено достаточное условие на параметры неоднородной модели Поттса на дереве Кэли, при котором неупорядоченная фаза является экстремальной.

\section{1. ВВЕДЕНИЕ}

Дерево Кэли $\Gamma^{k}=(V, L)$ порядка $k \geqslant 1$ есть бесконечное дерево, т.е. граф̆ без циклов, из каждой вершины которого выходит ровно $k+1$ ребер, где $V$ - множество вершин $\Gamma^{k}$ и $L-$ множество его ребер.

Эта решетка обладает тем свойством, что число вершин, посешаемых за $n$ шагов, растет экспоненциально с ростом $n$. Это более быстрый рост, чем $n^{d}$, независимо от значения $d$, где $n^{d}$ - объем ящика с ребром, равным $n$, в $d$-мерном пространстве. Поэтому деревья Кэли и модели, определяемые на нем, являются бесконечномерными [1].

Рассмотрим неоднородную модель Поттса, гамильтониан которой имеет вид

$$
H(\sigma)=-\sum_{\langle x, y\rangle \in L} J_{x y} \delta_{\sigma(x) \sigma(y)}
$$

где $J_{x y} \in \mathbb{R}, \sigma(x) \in \Phi=\left\{\sigma_{1}, \sigma_{2}, \ldots, \sigma_{q}\right\} \subset \mathbb{R}^{q-1}$,

$$
\sigma_{i} \sigma_{j}= \begin{cases}1, & \text { если } i=j \\ -\frac{1}{q-1}, & \text { если } i \neq j\end{cases}
$$

и $\delta$ - символ Кронекера.

Основная задача для данного гамильтониана - это описание множества всех предельных гиббсовских мер.

Известно, что множество всех гиббсовских мер с данным гамильтонианом является непустым, выпуклым, компактным подмножеством множества всех вероятностных мер [2]. Поэтому естественна задача изучения крайних гиббсовских мер. Эта задача

* Институт математики им. В.И. Романовского АН РУз, Ташкент, Республика Узбекистан 
весьма трудная, и более или менее окончательные результаты получаются лишь при малых значениях $\beta=1 / T$, где $T>0$ - температура.

В работах [3-7] построены крайние гиббсовские меры для моделей Изинга, Поттса и для некоторых обобщений этих моделей на дереве Кэли. В работе [3] доказано, что неупорядоченная фаза модели Изинга является крайней при $T \geqslant T_{\text {c }}$ и не является крайней при $T<T_{\mathrm{c}}$, где $T_{\mathrm{c}}$ - критическая температура модели спинового стекла.

В настоящей работе мы определим достаточное условие на параметры неоднородной модели Поттса, при котором неоднородная фаза является крайней.

\section{2. ОПРЕДЕЛЕНИЯ И ПРЕДВАРИТЕЛЬНЫЕ РЕЗУЛЬТАТЫ}

Из (2) следует, что для любых $x, y \in V$

$$
\delta_{\sigma(x) \sigma(y)}=\frac{q-1}{q}\left(\sigma(x) \sigma(y)+\frac{1}{q-1}\right) .
$$

Следовательно, $H(\sigma)$ имеет вид

$$
H(\sigma)=-\sum_{\langle x, y\rangle \in L} J_{x y}^{\prime} \sigma(x) \sigma(y)
$$

где $J_{x y}^{\prime}=(q-1) J_{x y} / q$. Введем обозначения

$$
W_{n}=\left\{x \in V \mid d\left(x, x^{0}\right)=n\right\}
$$

где $x^{0} \in V$ - фиксированная точка, $d(x, y)$ - число ребер кратчайшего пути, соединяющего $x$ и $y$,

$$
\begin{aligned}
& V_{n}=\bigcup_{m=1}^{n} W_{m}=\left\{x \in V \mid d\left(x, x^{0}\right) \leqslant n\right\}, \\
& L_{n}=\left\{l=\langle x, y\rangle \in L \mid x, y \in V_{n}\right\} .
\end{aligned}
$$

Для $A \subset V$ обозначим через $\Omega_{A}=\Phi^{A}$ множество конфигураций на $A$. Пусть $h_{x} \in$ $\mathbb{R}^{q-1}$ - векторнозначная функция $x \in V$. Рассмотрим для каждого $n$ меру $\mu_{n}$ на $\Omega_{V_{n}}$, определенную формулой

$$
\mu_{n}\left(\sigma_{n}\right)=Z_{n}^{-1} \exp \left\{\sum_{\langle x, y\rangle \in L_{n}} \beta J_{x y}^{\prime} \sigma(x) \sigma(y)+\sum_{x \in W_{n}} h_{x} \sigma(x)\right\}
$$

где $\sigma_{n}=\left\{\sigma(x), x \in V_{n}\right\} \in \Omega_{V_{n}}$ и $Z_{n}^{-1}$ - нормирующий множитель.

Условия согласованности для $\mu_{n}\left(\sigma_{n}\right), n \geqslant 1$, определяются равенством

$$
\sum_{\sigma^{(n)}} \mu_{n}\left(\sigma_{n-1}, \sigma^{(n)}\right)=\mu_{n-1}\left(\sigma_{n-1}\right)
$$

где $\sigma^{(n)}=\left\{\sigma(x), x \in W_{n}\right\}$. В качестве базиса в $\mathbb{R}^{q-1}$ берем $\sigma_{1}, \sigma_{2}, \ldots, \sigma_{q-1}$. 
Теорема 1. Меры $\mu_{n}\left(\sigma_{n}\right), n=1,2, \ldots$, удовлетворяют (5) тогда и только тогда, когда для векторов $h_{x} \in \mathbb{R}^{q-1}, x \in V$, вьполняется равенство

$$
h_{x}=\sum_{y \in S(x)} F\left(h_{y}, q, \theta_{x y}\right)
$$

əде $F: \mathbb{R}^{q-1} \rightarrow \mathbb{R}^{q-1}:$

$$
F_{i}=\ln \left[\frac{\left(\theta_{x y}-1\right) \exp h_{i}+\sum_{j=1}^{q-1} \exp h_{j}+1}{\sum_{j=1}^{q-1} \exp h_{j}+\theta_{x y}}\right],
$$

$\theta_{x y}=\exp \left(J_{x y}^{\prime} / T\right), \quad h=\left(h_{1}, \ldots, h_{q-1}\right), \quad F(h)=\left(F_{1}, \ldots, F_{q-1}\right), \quad S(x)=\{y \in V \mid$ $d(x, y)=1, \quad \exists$ пакое, что $\left.y \in W_{n}\right\}$.

ДокАЗАТЕЛЬСТво. Непосредственно подставляя (4) в (5), можно проверить, что (5) и (6) эквивалентны.

Легко проверить, что отображение $F$ удовлетворяет следующему свойству:

$$
\|F(h)\| \leqslant \frac{(q-1)(\theta-1)}{\theta}\|h\|
$$

где $h=\left(h_{1}, \ldots, h_{q-1}\right) \in \mathbb{R}^{q-1},\|h\|=\max _{1 \leqslant i \leqslant q-1}\left|h_{i}\right|$.

В силу теоремы Колмогорова из теоремы 1 следует, что каждой предельной гиббсовской мере $\mu$ на $\Omega_{V}$ соответствует совокупность векторов $\left\{h_{x}, x \in V\right\}$, которая удовлетворяет условию (6), и обратно, каждой совокупности векторов $\left\{h_{x}, x \in V\right\}$, удовлетворяюших (6), соответствует единственная предельная мера $\mu$ на $\Omega_{V}$.

Заметим, что независимо от значений параметров $k \geqslant 1, q \geqslant 2, \theta_{x y} \in \mathbb{R}, x, y \in V$, совокупность векторов $\left\{h_{x}=(0,0, \ldots, 0) \in \mathbb{R}^{q-1}, x \in V\right\}$ удовлетворяет уравнениям (6).

Обозначим через $\mu_{0}$ меру, соответствуюшую $\left\{h_{x}=(0,0, \ldots, 0) \in \mathbb{R}^{q-1}, x \in V\right\}$ по теореме 1. Эта мера называется неупорядоченной фазой неоднородной модели Поттса.

В этой работе мы докажем, что мера $\mu_{0}$ при некоторых условиях на параметры удовлетворяет следуюшему свойству.

Свойство $\mathrm{E}^{1)}$. Для любых $\varepsilon>0, \quad n>0$ иконфигураций $\sigma_{n}=\left\{\sigma(x), x \in V_{n}\right\} \in$ $\Omega_{V_{n}}$ существуют $N>n$ и $A_{N} \subset \Omega_{W_{N}}$ mакие, что:

1) $\mu_{0}\left(A_{N}\right)>1-\varepsilon$

2) $\left|\mu_{0}\left(\sigma_{n} \mid \sigma^{(N)}\right)-\mu_{0}\left(\sigma_{n}\right)\right|<\varepsilon \partial л я$ любого $\sigma^{(N)} \in A_{N}$.

Из свойства Е следует, что для типичных граничных условий $\sigma^{(N)}$ условное распределение $\mu_{0}\left(\sigma_{n} \mid \sigma^{(N)}\right)$ сходится к безусловному $\mu_{0}\left(\sigma_{n}\right)$ при $N \rightarrow \infty$ и мера $\mu_{0}$ является крайней (см. [2]) .

\footnotetext{
1) Extremity.
} 


\section{3. ОСНОВНЫЕ РЕЗУЛЬТАТЫ}

Ради краткости введем обозначения

$$
\begin{aligned}
\mu_{0}\left(\sigma_{n}\right) & =\mu_{0}\left(\left\{\sigma_{n}\right\} \times \Omega_{V \backslash V_{n}}\right), \\
\mu_{0}\left(\sigma_{n} \mid \sigma^{(N)}\right) & =\frac{\mu_{0}\left(\sigma_{n}, \sigma^{(N)}\right)}{\mu_{0}\left(\sigma^{(N)}\right)},
\end{aligned}
$$

где

$$
\mu_{0}\left(\sigma_{n}, \sigma^{(N)}\right)=\mu_{0}\left(\left\{\sigma_{n}\right\} \times\left\{\sigma^{(N)}\right\} \times \Omega_{V \backslash\left(V_{n} \cup W_{N}\right)}\right) .
$$

Подставляя $h_{x}=(0,0, \ldots, 0) \in \mathbb{R}^{q-1}, x \in W_{N}$, в (4), получим

$$
\mu_{0}\left(\sigma_{N}\right)=Z_{N}^{-1} \exp \left\{-\frac{1}{T} H_{N}\left(\sigma_{N}\right)\right\}
$$

где

$$
H_{N}\left(\sigma_{N}\right)=-\sum_{\langle x, y\rangle \in L_{N}} J_{x y}^{\prime} \sigma(x) \sigma(y) .
$$

Обозначим

$$
h_{x}^{(N)}=\frac{1}{T} \sum_{y \in S(x)} J_{x y}^{\prime} \sigma(y), \quad x \in W_{N-1},
$$

тогда

$$
H_{N}\left(\sigma_{N}\right)=H_{N-1}\left(\sigma_{N-1}\right)-T \sum_{x \in W_{N-1}} h_{x}^{(N)} \sigma(x) .
$$

Следовательно,

$$
\mu_{0}\left(\sigma_{N}\right)=Z_{N}^{-1} \exp \left\{-\frac{1}{T} H_{N-1}\left(\sigma_{N-1}\right)+\sum_{x \in W_{N-1}} h_{x}^{(N)} \sigma(x)\right\} .
$$

Пусть $\sigma(y)$ независимо принимает значения $\sigma(y)=\sigma_{i}, \sigma_{i} \in \Phi$, с вероятностью $1 / q$. Совместное распределение случайных векторов $\left\{\sigma(x), x \in V_{N-1}\right\}$ и $\left\{h_{x}^{(N)}, x \in W_{N-1}\right\}=$ $h^{(N, N-1)}$ относительно $\mu_{0}$ имеет вид

$$
\begin{gathered}
\mu_{0}\left(\sigma_{N-1}, h^{(N, N-1)}\right)=\sum_{\frac{1}{T} \sum_{y \in S(x)} J_{x y}^{\prime} \sigma(y)=h_{x}^{(N)}, x \in W_{N-1}} \mu_{0}\left(\sigma_{N}\right)= \\
=Z_{N}^{-1} \exp \left\{-\frac{1}{T} H_{N-1}\left(\sigma_{N-1}\right)+\sum_{x \in W_{N-1}} h_{x}^{(N)} \sigma(x)\right\} \prod_{x \in W_{N-1}} \nu\left(h_{x}^{(N)}\right),
\end{gathered}
$$

где $\nu\left(h_{x}^{(N)}\right)$ - распределение случайного вектора (9).

Формула (11) напоминает формулу (4), но здесь значение $h_{x}^{(N)}$ случайно. Применяя рекуррентные уравнения (6), получим

$$
h_{x}^{(N)}=\sum_{y \in S(x)} F\left(h_{y}^{(N)}, q, \theta_{x y}\right) .
$$


Используя $\left(6^{\prime}\right)$, определим множество случайных векторов $\left\{h_{x}^{(N)}=h_{x}^{(N)}\left(\sigma^{(N)}\right), \quad x \in\right.$ $\left.V_{N-1}\right\}$. Случайные векторы $h_{x}^{(N)}$ удовлетворяют условию $\left(6^{\prime}\right)$. Поэтому совместное распределение случайных векторов $\left\{\sigma(x), x \in V_{n}\right\}$ и $\left\{h_{x}^{(N)}, x \in W_{n}\right\}=h^{(N, n)}$ относительно $\mu_{0}$ имеет вид

$$
\mu_{0}\left(\sigma_{n}, h^{(N, n)}\right)=Z_{N, n}^{-1} \exp \left[-\frac{1}{T} H_{n}\left(\sigma_{n}\right)+\sum_{x \in W_{n}} h_{x}^{(N)} \sigma(x)\right] \prod_{x \in W_{n}} \nu_{N-n}\left(h_{x}^{(N)}\right)
$$

где $\nu_{N-n}\left(h_{x}^{(N)}\right)$ - распределение случайного вектора $h_{x}^{(N)}$ для $x \in W_{n}$.

Пусть $h=\left(h_{1}, \ldots, h_{q-1}\right) \in \mathbb{R}^{q-1}$ и

$$
\|h\|=\max _{1 \leqslant i \leqslant q-1}\left|h_{i}\right|
$$

Лемма 1. Для любого $x \in W_{n}, n \leqslant N-1$, имеет место равенство

$$
E h_{x i}^{(N)}=0, \quad i=1,2, \ldots, q-1,
$$

əде $h_{x}^{(N)}=\left(h_{x 1}^{(N)}, h_{x 2}^{(N)}, \ldots, h_{x(q-1)}^{(N)}\right) \in \mathbb{R}^{q-1}, \quad$ E-математическое ожсидание.

ДокАЗАТЕЛЬСтво. Пусть $x \in W_{N-1}$, тогда

$$
\begin{aligned}
E h_{x i}^{(N)}= & \sum_{\sigma^{(N)}} h_{x i}^{(N)}\left(\sigma^{(N)}\right) \mu\left(\sigma^{(N)}\right)= \\
& =\frac{1}{q^{(k+1) k^{N-1}}} \sum_{\sigma^{(N)}} h_{x i}^{(N)}\left(\sigma^{(N)}\right)=\frac{1}{T q^{(k+1) k^{N-1}}} \sum_{\sigma^{(N)}}\left(\sum_{y \in S(x)} J_{x y}^{\prime} \sigma^{(i)}(y)\right),
\end{aligned}
$$

где $\sigma(y)=\left(\sigma^{(1)}(y), \sigma^{(2)}(y), \ldots, \sigma^{(q-1)}(y)\right) \in \Phi, \quad \mu$ - полиномиальное распределение на $\Omega_{W_{N}}$.

Заметим, что для элементов множества $\Phi$ справедливо равенство

$$
\sum_{i=1}^{q} \sigma_{i}=0
$$

В силу (13) из (12) получим $E h_{x i}^{(N)}=0, x \in W_{N-1}, i=1,2, \ldots, q-1$.

Теперь докажем лемму в случае $x \in W_{n}, n<N-1$.

Заметим, что для $x \in W_{N-1}, \sigma^{(N)} \in \Omega_{W_{N}}$ вектор $h_{x}^{(N)}$ можно записать в виде

$$
\begin{aligned}
h_{x}^{(N)} & =\beta \sum_{y \in S(x)} J_{x y}^{\prime} \sigma(y)= \\
& =\beta \sum_{i=1}^{q} \alpha_{x i} \sigma_{i}=\left(\beta\left(\alpha_{x 1}-\alpha_{x q}\right), \beta\left(\alpha_{x 2}-\alpha_{x q}\right), \ldots, \beta\left(\alpha_{x(q-1)}-\alpha_{x q}\right)\right),
\end{aligned}
$$

где

$$
\alpha_{x i}=\sum_{y \in S(x): \sigma(y)=\sigma_{i}} J_{x y}^{\prime}, \quad i=1,2, \ldots, q
$$


Рассмотрим числа $A_{x, n}^{(i)}, x \in W_{n}, i=1,2, \ldots, q, n \leqslant N-1$, которые удовлетворяют следующим рекуррентным соотношениям:

$$
\begin{gathered}
A_{x, n}^{(i)}=\prod_{z \in S(x)}\left(\left(\theta_{x z}-1\right) A_{z, n+1}^{(i)}+\sum_{j=1}^{q} A_{z, n+1}^{(j)}\right), \quad x \in W_{n}, \quad i=1,2, \ldots, q, \quad n<N-1, \\
A_{y, N-1}^{(i)}=\exp \left(\beta \alpha_{y i}\right), \quad y \in W_{N-1} .
\end{gathered}
$$

Множество значений случайных величин $\alpha_{x i}=\alpha_{x i}\left(\sigma^{(N)}\right)$ не зависит от $i$. Отсюда следует, что множество значений случайных величин $A_{x, n}^{(i)}=A_{x, n}^{(i)}\left(\sigma^{(N)}\right)$ также не зависит от $i$.

Для доказательства леммы в случае $x \in W_{n}, n<N-1$, воспользуемся равенством $\left(6^{\prime}\right)$, получим

$$
\begin{aligned}
& E h_{x i}^{(N)}=E \sum_{z_{n+1} \in S(x)} F_{i}\left(h_{z_{n+1}}^{(N)} ; \theta_{x z_{n+1}}\right)= \\
& =\sum_{z_{n+1} \in S(x)} E F_{i}\left(\sum_{z_{n+2} \in S\left(z_{n+1}\right)} F(\cdots\right.
\end{aligned}
$$

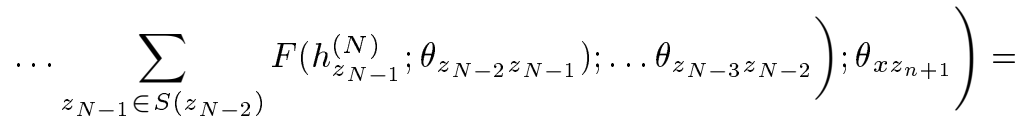

$$
\begin{aligned}
& =\sum_{z_{n+1} \in S(x)} E \ln \frac{\left(\theta_{x z_{n+1}}-1\right) A_{z_{n+1}, n+1}^{(i)}+\sum_{j=1}^{q} A_{z_{n+1}, n+1}^{(j)}}{\left(\theta_{x z_{n+1}}-1\right) A_{z_{n+1}, n+1}^{(q)}+\sum_{j=1}^{q} A_{z_{n+1}, n+1}^{(j)}}=\frac{1}{q^{(k+1) k^{N-1}}} \times \\
& \times \sum_{z_{n+1} \in S(x)} \ln \frac{\prod_{\sigma^{(N)}}\left[\left(\theta_{x z_{n+1}}-1\right) A_{z_{n+1}, n+1}^{(i)}+\sum_{j=1}^{q} A_{z_{n+1}, n+1}^{(j)}\right]}{\prod_{\sigma^{(N)}}\left[\left(\theta_{x z_{n+1}}-1\right) A_{z_{n+1}, n+1}^{(q)}+\sum_{j=1}^{q} A_{z_{n+1}, n+1}^{(j)}\right]} .
\end{aligned}
$$

Так как область значений $A_{z_{n+1}, n+1}^{(i)}$ не зависит от $i$, то из $(14)$ получим $E h_{x i}^{(N)}=0$, $x \in W_{n}$. Лемма доказана.

Пусть $D h_{x i}^{(N)}$ - дисперсия случайной величины $h_{x i}^{(N)}=h_{x i}^{(N)}\left(\sigma^{(N)}\right), i=1,2, \ldots, q-1$. Обозначим

$$
\begin{aligned}
& a(x)=\sum_{y \in S(x)}\left[(q-1) \frac{\theta_{x y}-1}{\theta_{x y}}\right]^{2}, \quad x \in V, \\
& b(x)=\sum_{y \in S(x)}\left|J_{x y}^{\prime}\right|, \quad x \in V .
\end{aligned}
$$

Пусть $a(x)$ удовлетворяет одному из следуюших условий.

УСловиЕ 1а. $a(x) \equiv 1$ для любого $x \in V$;

УСловиЕ 1б. Для любого $x \in V$ и любых бесконечных путей $\left\{x, x_{1}, x_{2}, \ldots\right\}$ выполняется равенство

$$
\prod_{i=1}^{\infty} a\left(x_{i}\right)=0 .
$$

Пусть $b(x)$ удовлетворяет следуюшему условию.

УсловиЕ 2 . Сушествует $M>0$ такое, что $b(x) \leqslant M$ для любого $x \in V$. 
ЛЕмма 2. Если выполняются условия 1а или 1 и и условие 2, то

$$
\lim _{N-n \rightarrow \infty} D h_{x i}^{(N)}=0, \quad x \in W_{n}, \quad i=1,2, \ldots, q-1 .
$$

ДокАЗАТЕЛЬСтво. Из уравнений $(6)$ в силу независимости $h_{x i}^{(N)}$ следует, что

$$
\begin{gathered}
D h_{x i}^{(N)}=\sum_{y \in S(x)} D F_{i}\left(h_{y i}^{(N)}, q, \theta_{x y}\right), \\
x \in W_{m-1}, \quad y \in W_{m}, \quad m \leqslant N-1 .
\end{gathered}
$$

В силу неравенства (7) имеем

$$
D h_{x i}^{(N)} \leqslant \sum_{y \in S(x)}\left(\frac{(q-1)\left(\theta_{x y}-1\right)}{\theta_{x y}}\right)^{2} D h_{y i}^{(N)} \leqslant a(x) D h_{y^{\prime} i}^{(N)}
$$

где

$$
D h_{y^{\prime} i}^{(N)}=\max _{y \in S(x)} D h_{y i}^{(N)} .
$$

Итерируя это неравенство для

$$
x \in W_{n}, \quad y \in W_{N-1}, \quad n \leqslant N-1,
$$

получим

$$
D h_{x i}^{(N)} \leqslant\left(\prod_{z: x<z \leqslant y} a(z)\right)^{N-n-1} D h_{y i}^{(N)}
$$

Из (18) при выполнении условия $1 б$ следует (15).

Теперь докажем, что (15) выполняется и при условии 1а. В этом случае из (17) получим

$$
D h_{x i}^{(N)} \leqslant D h_{y^{\prime} i}^{(N)}, \quad x \in W_{m-1}, \quad y^{\prime} \in W_{m} .
$$

Откуда следует, что $D h_{x i}^{(N)}$ не убывают при возрастании $m$. Положим, что

$$
\lim _{N-n \rightarrow \infty} D h_{x i}^{(N)}=\gamma>0, \quad x \in W_{n} .
$$

Заметим, что для $\|h\|>\varepsilon$ сушествует $\delta=\delta(\varepsilon)$ такое, что

$$
\|F(h)\| \leqslant(1-\delta) \frac{(q-1)(\theta-1)}{\theta}\|h\| .
$$

Таким образом, для $x \in W_{m-1}, y \in W_{m}$ мы имеем

$$
D h_{x i}^{(N)} \leqslant D h_{y i}^{(N)}\left(1-\frac{\delta D_{\varepsilon} h_{y i}^{(N)}}{D h_{y i}^{(N)}}\right)
$$


где

$$
D_{\varepsilon} t=\sum_{|t|>\varepsilon} t^{2} \nu_{N-m}(t)
$$

Из (9) и условия 2 вытекает

$$
\left|h_{y i}^{(N)}\right| \leqslant \beta \sum_{z \in S(y)}\left|J_{x y}^{\prime}\right|=\beta b(x) \leqslant \beta M
$$

для любого $x \in V_{N-1}$. Следовательно,

$$
D h_{x i}^{(N)} \leqslant D h_{y i}^{(N)}\left(1-\delta_{0} D_{\varepsilon} h_{y i}^{(N)}\right),
$$

где $\delta_{0}=\delta /(\beta M)^{2}$. Итерируя эту оценку, получим

$$
D h_{x i}^{(N)} \leqslant D h_{y i}^{(N)} \prod_{z: x<z \leqslant y}\left(1-\delta_{0} D_{\varepsilon} h_{z i}^{(N)}\right) .
$$

В силу (20) из последнего неравенства получим

$$
\lim _{N-n \rightarrow \infty} D_{\varepsilon}\left(h_{x i}^{(N)}\right)=0, \quad x \in W_{n} .
$$

Так как $\varepsilon>0$ произвольное, то отсюда следует (15) при условии 1а. Лемма доказана.

ЛЕмма 3. Если выполняются условия 1 и 2, то

$$
\operatorname{Prob}\left\{\lim _{N \rightarrow \infty}\left\|h_{x}^{(N)}\right\|=0, x \in W_{n}\right\}=1,
$$

әде п фиксировано.

ДоказАТЕЛьство. Рассмотрим неравенство Чебьшева

$$
\operatorname{Prob}\left\{\left|h_{x i}^{(N)}-E h_{x i}^{(N)}\right| \geqslant \varepsilon\right\} \leqslant \frac{D h_{x i}^{(N)}}{\varepsilon^{2}} .
$$

В силу лемм 1 и 2 из (22) имеем

$$
\lim _{N \rightarrow \infty} \operatorname{Prob}\left\{\left|h_{x i}^{(N)}\right|<\varepsilon\right\}=1, \quad i=1,2, \ldots, q-1 .
$$

Из (23) вытекает (21). Лемма доказана.

Теперь докажем, что $\mu_{0}$ удовлетворяет свойству Е при условиях 1 и 2 . Пусть $n>0$, $\delta>0$ фиксированы. Рассмотрим

$$
A_{N, \delta}=\left\{\sigma^{(N)}=\left\{\sigma(x), x \in W_{N}\right\} \mid\left\|h_{x}{ }^{(N)}\left(\sigma^{(N)}\right)\right\| \leqslant \delta, \quad x \in W_{n}\right\} .
$$

Докажем, что

$$
\lim _{N \rightarrow \infty} \mu_{0}\left(A_{N, \delta}\right)=1
$$

3 Теоретическая и математическая физика, т. 124, № 3, 2000 г. 
Из $\left(11^{\prime}\right)$ имеем

$$
\mu_{0}\left(A_{N, \delta}\right)=\frac{\sum_{\left\{\left\|h_{x}^{(N)}\right\| \leqslant \delta, x \in W_{n}\right\}} Q_{n}\left(\left\{h_{x}^{(N)}, x \in W_{n}\right\}\right)}{\sum_{\left\{h_{x}^{(N)}, x \in W_{n}\right\}} Q_{n}\left(\left\{h_{x}^{(N)}, x \in W_{n}\right\}\right)}
$$

где

$$
Q_{n}\left(\left\{h_{x}^{(N)}, x \in W_{n}\right\}\right)=\sum_{\sigma_{n}} \exp \left\{-\frac{1}{T} H_{n}\left(\sigma_{n}\right)+\sum_{x \in W_{n}} h_{x}^{(N)} \sigma(x)\right\} \prod_{x \in W_{n}} \nu_{N-n}\left(h_{x}^{(N)}\right) .
$$

В силу леммы 3 равенство (24) следует из (25).

Оценим теперь величину $\left|\mu_{0}\left(\sigma_{n} \mid \sigma^{(N)}\right)-\mu_{0}\left(\sigma_{n}\right)\right|$. Из $\left(11^{\prime}\right)$ получаем

$$
\mu_{0}\left(\sigma_{n} \mid \sigma^{(N)}\right)=\frac{\exp \left\{-\frac{1}{T} H_{n}\left(\sigma_{n}\right)+\sum_{x \in W_{n}} h_{x}^{(N)} \sigma(x)\right\}}{\sum_{\sigma_{n}} \exp \left\{-\frac{1}{T} H_{n}\left(\sigma_{n}\right)+\sum_{x \in W_{n}} h_{x}^{(N)} \sigma(x)\right\}}
$$

где $h_{x}^{(N)}=h_{x}^{(N)}\left(\sigma^{(N)}\right), \quad x \in W_{n}$, определяется по формулам $(9)$ и $\left(6^{\prime}\right)$. Более того, из (8) следует

$$
\mu_{0}\left(\sigma_{n}\right)=\frac{\exp \left\{-\frac{1}{T} H_{n}\left(\sigma_{n}\right)\right\}}{\sum_{\sigma_{n}} \exp \left\{-\frac{1}{T} H_{n}\left(\sigma_{n}\right)\right\}}
$$

Так как $n$ фиксировано, из соотношений (26) и (27) вытекает, что для любого $\varepsilon>0$ существует $\delta>0$ такое, что

$$
\left|\mu_{0}\left(\sigma_{n} \mid \sigma^{(N)}\right)-\mu_{0}\left(\sigma_{n}\right)\right|<\varepsilon,
$$

где $\left\|h_{x}^{(N)}\right\| \leqslant \delta$ для всех $x \in W_{n}$, т.е. для $\sigma^{(N)} \in A_{N, \delta}$.

Следовательно, $\mu_{0}$ удовлетворяет свойству Е. Таким образом, доказана следуюшая теорема.

ТЕОРЕМа 2. Если выполняются условия 1 и 2, то неупорядоченная фаза неоднородной модели Поттса на дереве Кәли является крайней.

Благодарности. Авторы выражают глубокую признательность проф. Н. Н. Ганиходжаеву за внимание к работе.

\section{Список литературы}

[1] Р. Бәкстер. Точно решаемые модели в статистической механике. М.: Мир, 1985.

[2] Я.Г. Синай. Теория фазовых переходов: Строгие результаты. М.: Наука, 1980.

[3] P. M. Bleher. Commun. Math. Phys. 1990. V. 128. P. 411-419.

[4] П. М. Блехер, Н. Н. Ганиходжаев. Теор. вероятн. и ее примен. 1990. Т. 35. № 2. С. 220-230.

[5] Н. Н. Ганиходжаев. ДАН РУз. 1992. № 6-7. С. 4-7.

[6] Н.Н. Ганиходжаев, У. А. Розиков. ТМФ. 1997. Т. 111. № 1. С. 109-117.

[7] Н. Н. Ганиходжаев, У. А. Розиков. Матем. сб. 1999. Т. 190. № 2. С. 31-42.

Поступила в редакцию 2.II.2000 г. 